\title{
Plasma viscosity in inflammatory bowel disease
}

\author{
A J Lobo, S C Jones, L D Juby, A T R Axon
}

\begin{abstract}
Aims: To assess the relation of plasma viscosity to disease activity in patients with inflammatory bowel disease.

Methods: Crohn's disease $(n=60)$ and ulcerative colitis $(n=71)$ were diagnosed on the basis of typical histological or radiological features. Active Crohn's disease was defined as a Crohn's disease activity index of 150 or over. Active ulcerative colitis was defined as a liquid stool passed three times a day or more with blood. Blood samples were assessed for haemoglobin concentration, total white cell count, platelets, plasma viscosity, erythrocyte sedimentation rate, serum albumin, and C-reactive protein.

Results: Plasma viscosity was higher in those with active Crohn's disease compared with those with inactive Crohn's disease or active ulcerative colitis. Plasma viscosity correlated significantly with erythrocyte sedimentation rate, Creactive protein, and platelet count in patients with Crohn's disease. In ulcerative colitis plasma viscosity correlated only with serum C-reactive protein. Plasma viscosity showed a low sensitivity for detecting active Crohn's disease, with $48 \%$ of those with active disease having a plasma viscosity within the laboratory reference range.

Conclusions: Plasma viscosity is related to disease activity in Crohn's disease, but is insufficiently sensitive for it to replace erythrocyte sedimentation rate as a measure of the acute phase response in Crohn's disease.
\end{abstract}

Plasma viscosity can be used as a non-specific measure of the acute phase response ${ }^{12}$ and may have several advantages over the more widely used erythrocyte sedimentation rate (ESR). ${ }^{2-4}$

The first clinical use of plasma viscosity measurement was described in 1942, when its use in pulmonary tuberculosis was described, ${ }^{5}$ and an association between red cell sedimentation velocity and plasma viscosity was noted. The widespread use of plasma viscosity measurement, however, was delayed until an automated system for its measurement became available. One such commercially available system has been shown to be reliable and precise $^{3}$ and detailed guidelines for the assessment of the acute phase response, including the role of plasma viscosity, have been published. ${ }^{2}$
The advantages of plasma viscosity are that, unlike the ESR, its value is independent of packed cell volume, sex, and for practical purposes, age. ${ }^{6}$ Viscosity is lower in neonates ${ }^{7}$ and rises slightly in the elderly due to changes in the plasma fibrinogen concentration. ${ }^{8}$ The use of automated systems reduces the biohazard risks and produces reproducible, precise results and these can subjected to quality assurance. Plasma must be separated as soon as possible and never more than six hours after venesection, but once separated, its viscosity may remain stable for several days if kept at $15-20^{\circ} \mathrm{C}^{2}$

The initial capital cost of an automated viscometer is currently about $£ 7500$ with very low running costs. Measurement of ESR requires a very low initial outlay, but the subsequent running costs are higher. If the annual running costs are said to include laboratory disposables, appropriate venesection containers, a maintenance contract for the viscometer cost, interest repayments on the initial outlay for the viscometer and a sum to contribute to its eventual replacement, the approximate respective costs for performing 32000 (the annual number performed at this institution in 1990) measurements of ESR and plasma viscosity would be $£ 10848$ and $£ 6464$, respectively (P Day, personal communication). The initial capital outlay would therefore be recouped within two years and more rapidly if plasma viscosity was measured from the same blood container as the blood count.

There are few data on the use of plasma viscosity in clinical practice. In unselected patients attending a general medical and rheumatological clinic plasma viscosity was a more reliable measure of changes in acute phase reactants. ${ }^{4}$ In rheumatoid arthritis plasma viscosity was said to be at least as reliable as ESR and C-reactive protein (CRP) in terms of diagnosis, and as an index of improvement.9 More recently however, plasma viscosity was found to compare less well than ESR with a battery of other measures of inflammation in rheumatoid arthritis. ${ }^{10}$

There are no previous reports of the use of plasma viscosity in inflammatory bowel disease. This paper reports the prospective evaluation of plasma viscosity measurement in a large series of patients with inflammatory bowel disease.

\section{Methods}

One hundred and thirty one patients with inflammatory bowel disease attending the gas- 
trointestinal service of the General Infirmary, Leeds, were studied. Crohn's disease and ulcerative colitis were diagnosed on the basis of typical histological or radiological features. Sixty had Crohn's disease. This affected the small bowel in $19(32 \%)$, the large bowel in 22 $(37 \%)$, and both small and large bowel in 19 $(32 \%)$. Seventy one had ulcerative colitis and the extent was proctitis or proctosigmoiditis in $39(55 \%)$. Left sided disease (extending not further than the splenic flexure) in $12(17 \%)$, and subtotal or total colitis in $17(24 \%)$. In three patients the precise extent was not known but had been documented to extend to at least the splenic flexure.

\section{CLINICAL ASSESSMENT OF DISEASE ACTIVITY}

Active Crohn's disease was defined as a Crohn's disease activity index ${ }^{11}$ (CDAI) of 150 or over. The CDAI is a widely used clinical index in which the number of liquid stools per day, general well-being, and abdominal pain over the previous week are scored and weighted. The presence of the following clinical features also contribute to the score: iritis, aphthous ulceration of the mouth, arthritis or arthralgia, erythema nodosum, pyoderma gangrenosum, fever, abdominal mass, perianal disease or fistula elsewhere. Use of lomotil or opiates, percentage below standard bodyweight, and haematocrit also contribute to the final score.

Active ulcerative colitis was defined as a liquid stool passed three times a day or more with blood. The severity of active ulcerative colitis was assessed by the criteria of Truelove and Witts. ${ }^{12}$ By these criteria patients with a bowel frequency of four motions a day or less, and no more than small amounts of macroscopic blood in the stool are considered to have mild disease; six or more motions a day with macroscopic blood and one or more of: fever on two days out of four, tachycardia (mean pulse rate greater than 90 beats $/ \mathrm{min}$ ), anaemia (haemoglobin concentration below $10.5 \mathrm{~g} / \mathrm{l}$ ) or ESR greater than $30 \mathrm{~mm}$ in one hour, are considered to have severe disease. Moderate severity is attributed to attacks intermediate between mild and severe.

\section{LABORATORY ASSESSMENT OF DISEASE ACTIVITY} All patients had blood drawn for haemoglobin concentration, total white cell count, platelet count (Coulter counter; Coulter electronics, Luton), plasma viscosity (Coulter viscometer), ESR (Westergren method), C-reactive protein (CRP) (Boehring nephelometer; Hoechst UK, Hounslow), and serum albumin (sequential multiple autoanalyser with computer; Technicon, Basingstoke). The laboratory reference range for plasma viscosity was $1 \cdot 5-1 \cdot 72$ millipascal seconds ( $\mathrm{mPa} . \mathrm{s}$ ), and for C-reactive protein, the lower limit of detection of the assay is $5 \mathrm{mg} / 1$ and the reference range is $5-10 \mathrm{mg} / 1$.

Differences between plasma viscosity in active and inactive disease and between Crohn's disease and ulcerative colitis were assessed by the Mann-Whitney U-test. The correlation between plasma viscosity and the CDAI, ulcerative colitis disease severity and laboratory variables was performed using the Spearman rank order correlation coefficient.

\section{Results}

By the criteria defined above, 21 patients had active Crohn's disease, 39 had inactive disease; 24 had active ulcerative colitis (11 mild, 10 moderate, three severe) and 47 had inactive disease.

The plasma viscosity was significantly higher in those with active Crohn's disease (median $1.73 \mathrm{mPa}$.s; range $1.50-2.04$ ) than in those with inactive disease (median $1.62 \mathrm{mPa} . \mathrm{s}$; range 1.5-1.92 (figure)). There was a large overlap, however, between the two groups, and 10 of 21 $(48 \%)$ patients with active disease had values within the reference range. In eight of these this normal plasma viscosity was associated with an increased CRP (median $77.5 \mathrm{mg} / \mathrm{l}$; range 23-145), and in two the CRP was not increased.

Plasma viscosity was also higher in patients with active Crohn's disease compared with those with active ulcerative colitis (median 1.65 mPa.s; range 1.5-1.92). There was a trend towards increasing plasma viscosity with increasing disease severity in ulcerative colitis but this was not significant $(r=0 \cdot 22 ; p=0.07)$. In patients with ulcerative colitis plasma viscosity correlated with $\mathrm{C}$-reactive protein $(r=0.25 ; p=0.03)$, but not with any other laboratory measurement.

In Crohn's disease plasma viscosity showed a significant correlation with the ESR (Spearman $r=0.77, p=0.00001), \operatorname{CDAI}(r=0.34$, $\mathrm{p}=0.009), \operatorname{CRP}(\mathrm{r}=0.44, \mathrm{p}=0.001)$, platelet count $(r=0.43, p=0.001)$, and correlated negatively with albumin $(r=-0.44$, $p=0.002)$. The inverse correlation with haemoglobin did not reach significance $(\mathrm{r}=-0.23, \mathrm{p}=0.07)$.

For comparison, the ESR values according to disease activity are shown alongside the plasma viscosity values in fig $1 \mathrm{~A}$ and the CRP values are shown in fig $1 \mathrm{~B}$.

The sensitivity and specificity of ESR and plasma viscosity in distinguishing active from inactive Crohn's disease depends on the value taken as a cut-off point. Examples are given in the table.

\section{Discussion}

Despite the claimed advantages of plasma viscosity over ESR, our data highlight limitations in the use of this test in inflammatory bowel disease. The assessment of disease activity in Crohn's disease is difficult, and a major clinical problem is to differentiate those with symptoms due to active disease from those with symptoms due to other causes. Laboratory indices may be particularly helpful here, but no laboratory test or clinical assessment has been shown to be without limitations. No gold standard exists for the assessment of disease activity in Crohn's disease. The most commonly used evaluation for clinical studies, including therapeutic trials, is the CDAI, which is closely related to physicians' overall assessment of disease severity. ${ }^{11}$

Forty eight percent of those with active Crohn's disease had plasma viscosity values within the reference range. The presence of symptoms not due to active disease may 

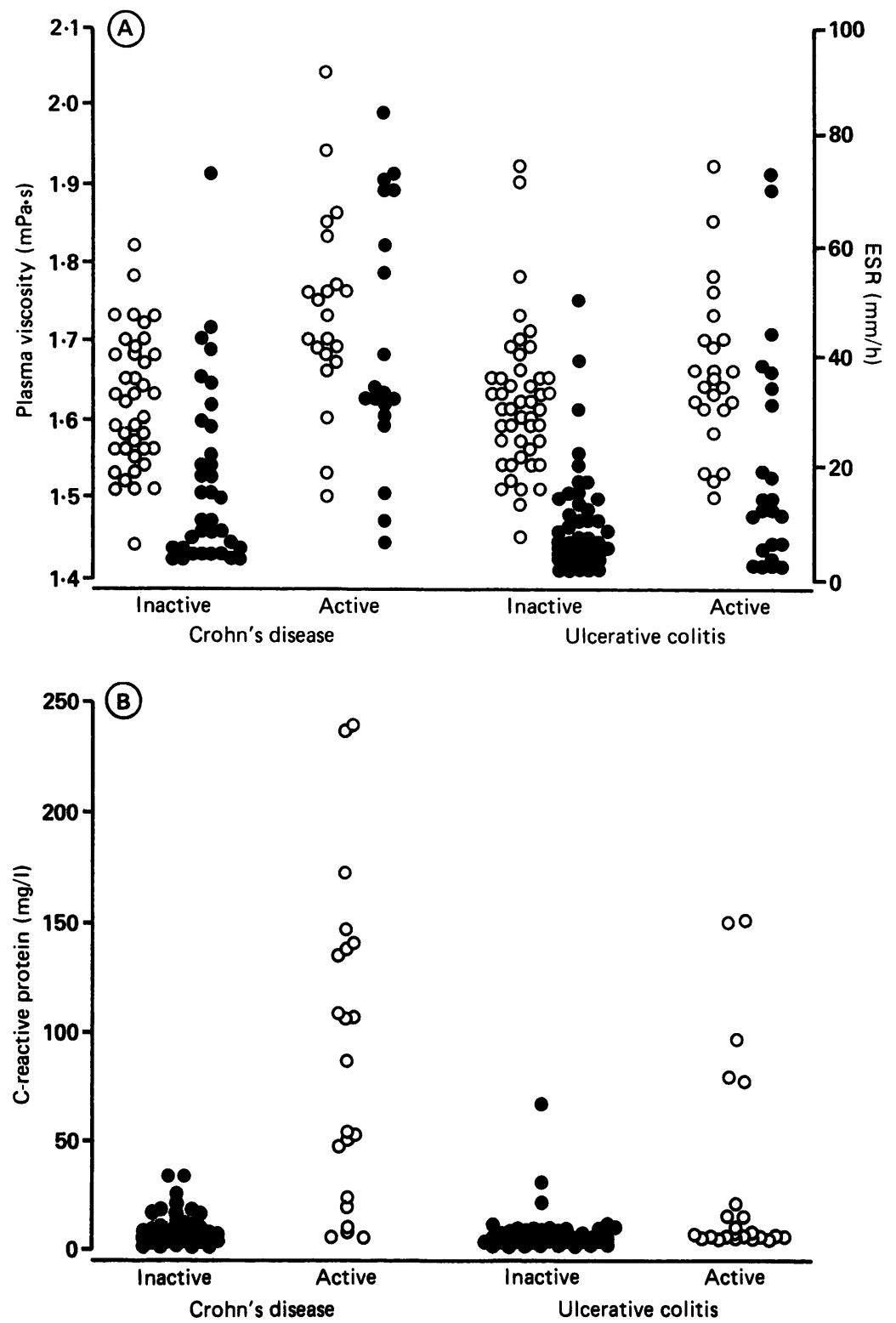

Figure (A) Plasma viscosity ( ) and erythrocyte sedimentation rate (ESR) (O) in active and inactive Crohn's disease and ulcerative colitis. (B) Serum $C$-reactive protein in active and inactive Crohn's disease and ulcerative colitis.
Sensitivity and specificity of plasma viscosity ( $P V)$ and ESR in distinguishing active from inactive Crohn's disease for different levels of both $P V$ and $E S R$

\begin{tabular}{lll}
\hline & Sensitivity (\%) & Specificity (\%) \\
\hline Plasma viscosity & & \\
$1.65 \mathrm{mPa} . \mathrm{s}$ & 90 & 67 \\
$1.72 \mathrm{mPa} . \mathrm{s}$ & 52 & 90 \\
ESR & & \\
$14 \mathrm{~mm} / \mathrm{h}$ & 90 & 54 \\
$20 \mathrm{~mm} / \mathrm{h}$ & 85 & 71 \\
$42 \mathrm{~mm} / \mathrm{h}$ & 35 & 90 \\
\hline
\end{tabular}

spuriously increase the CDAI, but eight of 10 patients with a normal plasma viscosity and high CDAI, also had increased concentrations of circulating CRP suggesting that the symptoms causing a high CDAI were genuinely due to active inflammation rather than the fibrotic strictures that complicate Crohn's disease or coexisting irritable bowel symptoms. This overlap between active Crohn's disease and both inactive disease and the reference range makes a single normal value a poor predictor for differentiating active from inactive disease. There is also an overlap of values for ESR between active and inactive Crohn's disease. In contrast, however, this is due to several increased values in patients without symptoms, and in the clinical context does not pose a diagnostic problem.

This can also be expressed in terms of the sensitivity and specificity of the tests. These are, in turn, critically dependent on where the cutoff lines are drawn. The upper limit of the reference range in our laboratory is $1.72 \mathrm{mPa}$.s. This gives a specificity of $90 \%$ but a sensitivity of just $52 \%$. In comparison, using an ESR of 20 as an upper limit, the sensitivity is much improved at $85 \%$, but with a lower specificity of $71 \%$. To obtain similar figures for the plasma viscosity would require the upper limit of the reference range to be dropped to $1.65 \mathrm{mPa}$.s. At the levels currently used therefore, the ESR is more sensitive at detecting active Crohn's disease, but the plasma viscosity is more specific.

It has been pointed out that plasma viscosity may be less useful in conditions associated with anaemia and hypoalbuminaemia as both tend to increase the sedimentation rate. $^{2}$ Crohn's disease is associated with both, although the hypoalbuminaemia may be partly due to the acute phase response. The anaemia of Crohn's disease may be multifactorial, but is partly related to disease activity, and the effect of an associated anaemia in increasing the sedimentation rate may make the ESR more sensitive. It is therefore of interest that in patients with Crohn's disease there was only a trend towards correlation between haemoglobin concentration and plasma viscosity. This presumably reflects a balance between the lack of effect of packed cell volume on viscosity and the common effect of disease activity by haemoglobin via the plasma protein concentration). The ESR has also been noted to perform better than plasma viscosity in rheumatoid arthritis, where similar considerations obtain. ${ }^{10}$

The higher values of plasma viscosity found in those with active Crohn's disease compared with active ulcerative colitis again reflect the acknowledged difference in the extent of the acute phase response between the two conditions. ${ }^{1314}$ Although there was a trend towards increasing plasma viscosity with increasing severity in ulcerative colitis, this was not significant, but may reflect the low number of cases in the severe group, which are now quite uncommon.

Our data cannot support the replacement of ESR by plasma viscosity for the assessment of inflammatory bowel disease, especially Crohn's disease. We have documented a considerable overlap in plasma viscosity between those with active and those with inactive disease. Although plasma viscosity may provide a greater specificity in the differentiation of active from inactive Crohn's disease, ESR is more sensitive, and in the clinical context this is more useful. 
1 Stuart J, Lewis SM. Monitoring the acute phase response (editorial). Br Med J 1988;297:1143-4.

2 International Committee for Standardization on Haematology (Expert Panel on Blood Rheology) Guidelines on the selection of laboratory tests for monitoring the acute phase response. J Clin Pathol 1988;41: 1203-12.

3 Cooke BM, Stuart J. Automated measurement of plasma viscosity by capillary viscometer. J Clin Pathol 1988; 41:1213-16.

4 Hutchinson RM, Eastham RD. Comparison of the erythrocyte sedimentation rate and plasma viscosity in erythrocyte sedimentation rate and plasma viscosity in detecting changes

5 Miller AK, Whittington RB. Plasma viscosity in pulmonary tuberculosis. Lancet 1942;ii:510-11.

6 Jung F, Roggenkamp HG, Ringelstein EB, et al. Effect of sex age, body weight, and smoking on plasma viscosity. Klin Wochenschr 1986;64:1076-81.

7 Linderkamp O. Blood rheology in the newborn infant. Clin Haematol 1987;1:801-25.
8 Meade TW, Chakrabati R, Haines AP, North WRS, Stirling Y. Characteristics infecting fibrinolytic activity and plasm fibrinogen concentrations. Br Med J 1979;1:153-6.

9 Pickup ME, Dixon JS, Hallett C, Bird HA, Wright V. Plasma viscosity - a new appraisal of its use as an index of disease activity in rheumatoid arthritis. Ann Rheum Dis 1981;40:272-5.

10 Bull BS, Westengard JC, Farr M, Bacon PA, Meyer PJ, Stuart J. Efficacy of tests used to monitor rheumatoid arthritis. Lancet 1989;ii:965-7.

11 Best WR, Becktel JM, Singleton JW, Kern F. Development of a Crohn's disease activity index. National co-operative of a Crohn's disease activity index. National co-operative

12 Truelove SC, Witts LJ. Cortisone in ulcerative colitis. Final report on a therapeutic trial. Br Med J 1955;2:1041-8.

13 Fagan EA, Dyck RF, Maton PN, Hodgson HJF, Chadwick VS, Pepys MB. Serum levels of C-reactive protein in Crohn's disease and ulcerative colitis. Eur J Clin Invest 1982;12:351-60.

14 Saverymuttu SH, Hodgson HJF, Chadwick VS, Pepys MB Differing acute phase responses in Crohn's disease and ulcerative colitis. Gut 1986;27:809-13. 